\title{
POST-LAUNCH CALIBRATION SUPPORT FOR VIIRS ONBOARD NASA NPP SPACECRAFT
}

\author{
Xiaoxiong Xiong ${ }^{1}$, Kwo-Fu Chiang ${ }^{2}$, Jeffrey McIntire ${ }^{2}$, Mathew Schwaller ${ }^{1}$, and James Butler ${ }^{1}$ \\ ${ }^{1}$ Sciences and Exploration Directorate, NASA/GSFC, Greenbelt, MD 20771, USA \\ ${ }^{2}$ Sigma Space Corp., 4801 Forbes Boulevard, Lanham, MD 20706, USA
}

\begin{abstract}
The NPP Instrument Calibration Support Element (NICSE) is one of the elements within the NASA NPP Science Data Segment (SDS). The primary responsibility of NICSE is to independently monitor and evaluate on-orbit radiometric and geometric performance of the Visible Infrared Imaging Radiometer Suite (VIIRS) instrument and to validate its Sensor Data Record (SDR) [1]. The NICSE interacts and works closely with other SDS Product Evaluation and Analysis Tools Elements (PEATE) and the NPP Science Team (ST) and supports their on-orbit data product calibration and validation efforts. The NICSE also works closely with the NPP Instrument Calibration Support Team (NICST) during sensor pre-launch testing in ambient and thermal vacuum environment [2]. This paper provides an overview of NICSE VIIRS sensor post-launch calibration support with a focus on the use of sensor on-board calibrators (OBC) for the radiometric calibration and characterization. It presents the current status of NICSE post-launch radiometric calibration tool development effort based on its design requirements.
\end{abstract}

\section{INTRODUCTION}

The NPOESS Preparatory Project (NPP) was developed as a risk reduction and bridge mission between the NASA Earth Observing System (EOS) and the National Polar-orbiting Operational Environmental Satellite System (NPOESS). The NPOESS program has recently been reconstructed and transitioned to the Joint Polar Satellite System (JPSS) and the Defense Weather Satellite System (DWSS). VIIRS is a key instrument on-board the NPP spacecraft, which is scheduled for launch in October 2011. Currently, all five flight instruments for the NPP mission have been fully integrated into the observatory, undergoing through various spacecraft-level testing. Along with VIIRS, the instruments are the Cross-track Infrared Sounder (CrIS), Ozone Mapping and Profiler Suite (OMPS), Clouds and the Earth Radiant Energy System (CERES), and the Advanced Technology Microwave Sounder (ATMS). To a large extent, the VIIRS instrument was developed from the Moderate Resolution Imaging Spectroradiometer (MODIS), one of the key instruments for the NASA EOS Terra and Aqua missions, with improvements made in a number of areas. VIIRS collects data in 22 spectral bands with wavelengths from 0.41 to $12 \mu \mathrm{m}$ and provides daily global observations of the land, ocean, and atmospheric parameters. To date, Terra MODIS has successfully operated for more than 11 years and Aqua MODIS for more than 9 years. Extensive on-orbit calibration and characterization activities have been implemented for each instrument. As a follow-on instrument for the NASA EOS MODIS, VIIRS has significantly benefitted from MODIS experience [3]. In addition to the lessons learned from MODIS pre-launch calibration and characterization activities, many of its on-orbit operation and calibration approaches have been considered and planned for VIIRS, including lunar observations and spacecraft maneuvers designed to support its on-orbit calibration.

Based on the experience and lessons from MODIS on-orbit operation and various calibration activities, NICSE has planned and developed a number of tasks to support the NPP VIIRS on-orbit calibration and characterization [4]. Examples from MODIS data are provided in this paper to demonstrate the use of sensor performance monitoring tools.

\section{PRE-LAUNCH SENSOR CHARACTERIZATION}

The VIIRS flight model unit 1 (F1) sensor has undergone through a comprehensive set of pre-launch calibration and characterization activities. The ambient phase testing was conducted from June 2007 to June 2008 at Raytheon's Santa Barbara Remote Sensing (SBRS) facility. Afterwards, the sensor was transported to Raytheon's El Segundo facility for sensor level thermal vacuum testing in the summer of 2009. In January 2010, the VIIRS F1 sensor was shipped to Ball Aerospace and Technology Corporation (BATC) in Boulder for integration, with other instruments, into the NPP spacecraft. Subsequently, performance testing was performed under ambient conditions from February 2010 to January 2011. From early March to late April of 2011, the NPP spacecraft successfully completed a series of tests under thermal vacuum conditions. After completing the remaining post thermal vacuum testing activities, the 
spacecraft will be transported to Vandenberg AFB and is scheduled for launch in October 2011.

All the pre-launch tests have been carefully designed prior to testing. A large amount of functional and radiometric calibration test data sets have been extensively analyzed by NASA NPP teams, including both NICST and NICSE. The analysis results have been documented in technical reports or memorandums for future reference purposes.

\section{ON-ORBIT CALIBRATION AND CHARACTERIZATION ACTIVITIES}

Similar to MODIS, VIIRS carries a set of on-board calibrators (OBC), which include a solar diffuser (SD) and a solar diffuser stability monitor (SDSM) for its reflective solar bands (RSB) calibration and a blackbody (BB) for the thermal emissive bands (TEB) calibration. Although the solar diffuser collects data every orbit, the SDSM, designed to track on-orbit changes in SD bi-directional reflectance factor (BRF), is scheduled only on a regular basis. The combined SD and SDSM calibration data provide absolute radiometric calibration for RSB at the sensor level. The instrument on-board blackbody is nominally controlled at a fixed temperature to provide information needed for TEB scan-to-scan radiometric calibration algorithm. Periodically, a sequence of warm-up and cool-down (WUCD) operations, which vary the $\mathrm{BB}$ temperature from instrument ambient to $315 \mathrm{~K}$, will be carried out over its entire mission in order to evaluate and maintain the TEB calibration and data quality. As expected, the designs and functions of VIIRS OBC are very similar to that of MODIS.

In addition to regular observations made by the VIIRS OBC, a number of special activities that involve spacecraft maneuvers, such as roll, yaw, and pitch maneuvers, have been planned and evaluated for their on-orbit implementation. These activities are designed and developed based on the experience from heritage sensors as an effort to further improve sensor characterization and calibration quality.

According to NPP flight operation design, VIIRS will be scheduled on a near monthly basis to view the Moon using the same strategy developed for MODIS lunar observations. As an effort to support this activity, a special VIIRS lunar view planner tool has been developed from MODIS experience. Figure 1 displays two recent Moon images from the Aqua MODIS RSB Band $1(0.65 \mu \mathrm{m}$; nadir resolution 250m) in 2011 (March and April). These data are collected through the instrument Space View (SV) port during regularly scheduled lunar roll maneuvers. The timing of the lunar observations and spacecraft roll angles are predicted by the lunar planner tool developed by the MODIS Characterization Support Team (MCST). A similar tool is being adopted by MCST and NICSE analysts for future
VIIRS lunar observations. Results from lunar view data analysis will provide an assessment of sensor radiometric calibration stability [5].
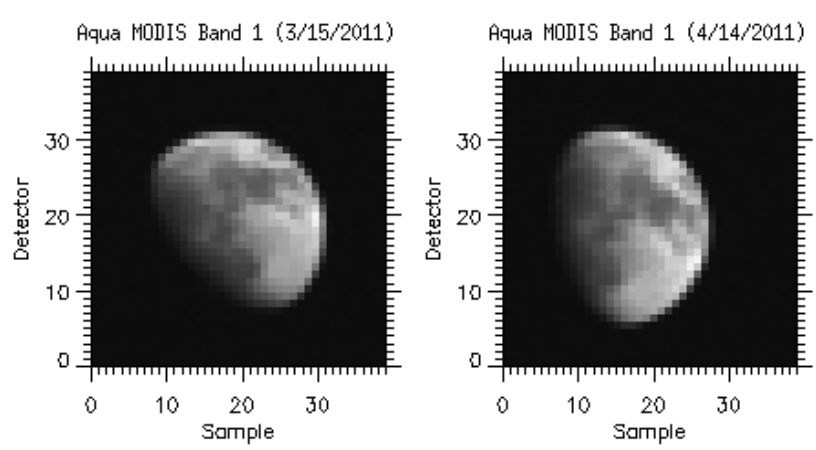

Fig. 1 Consecutive Aqua MODIS monthly lunar images were observed by Band $1(0.65 \mu \mathrm{m})$ in March and April 2011.

\section{TOOL DEVELOPMENT STATUS}

MODIS lessons and the experiences transferred from MCST at NASA Goddard Space Flight Center (GSFC) have significantly helped NICSE plan and develop a large number of post-launch tools for VIIRS on-orbit radiometric calibration and characterization. These new tools include algorithms and functions that are commonly used for MODIS. The VIIRS tools can be categorized in five areas described below.

\subsection{Input and output tools}

The VIIRS SDR operational code ingests the sensor Raw Data Record (RDR) and produces SDR and OBC intermediate product (OBC-IP) for every granule. The SDR contains calibrated Earth view data of each spectral band while the OBC-IP contains telemetry data and detector raw response from the internal on-board calibrators, which include the SD, SDSM, BB, and SV. The OBC-IP reader opens and reads the data in HDF format. It produces the output to be used for the OBC calibration tools. An image visualization tool was developed to display the SDR and perform certain statistical analysis on the SDR. Another tool was also developed to read the Telemetry RDR for trending purposes. In addition to data readers, NICSE is required to have the ability to modify the SDR calibration Look-UpTables (LUTs) used by the NPP data production system. The LUTs change tool can alter the array dimension and modify the content of each LUT.

\subsection{OBC calibration tools}

Radiometric calibration support is one of the important tasks in the NICSE design requirement. Each VIIRS detector (TEB and RSB) will be calibrated on-orbit using the OBC. Separate tools have been developed to compute detector 
calibration coefficients using the SD/SDSM for the RSB and the BB (during WUCD) for the TEB. The tools also compute each detector's signal to noise ratio (SNR) and other quality assurance characteristics. The algorithms used in the VIIRS SDSM calibration tool are similar to those for the MODIS instrument, likewise for the SDSM planner tool which can predict the calibration time periods in order to command SDSM operation in advance.

\subsection{Lunar calibration tools}

Lunar observations will also be used to support the VIIRS instrument calibration. Because of this, a lunar view planner tool has been developed, based on the MODIS lunar planner, to predict the lunar view observation opportunity. This planner tool predicts when and where the Moon will appear with respect to NPP orbit and VIIRS geometry, and at what phase angle. Such prediction can provide an accurate spacecraft roll maneuver schedule to both the spacecraft and instrument operation teams. NICSE requires the ability to use lunar view data to assess the absolute and relative radiometric response of VIIRS RSB detectors. The analysis of lunar view data, which is not part of the SDR operational production code, will be performed offline. Figure 1 shows examples of Aqua MODIS lunar observation. The results of the analysis will provide useful sensor characterization, including detector response comparison with respect to the SD calibration, relative spatial calibration of band-to-band registration (BBR), detector gain ratios, and other characteristics.

\subsection{RVS characterization tools}

The Response Versus Scan angle (RVS) of the VIIRS Half Angle Mirror (HAM) has been measured during pre-launch testing. On orbit, the RVS of the VIIRS HAM is not expected to degrade and change as much as the MODIS Scan Mirror. However, similar tools that can track the RVS change through the Earth view sector have been considered and developed based on MODIS experiences. For the RSB, analyzing the Earth view SDR data is the primary method for tracking the change of the relative RVS on-orbit. SDRs over relatively uniform ground targets will be selected for RSB RVS trending and characterization. For the TEB, the RVS can be measured again on-orbit using the dark Deep Space Maneuver (DSM) observations through the nadir Earth view port during the NPP pitch maneuver. A TEB RVS tool has been developed to facilitate offline calibration with respect to deep space at various scan angles on both HAM sides. By using these tools during post-launch operation, NICSE can characterize the HAM RVS and update the RVS LUTs for potential improvements of the SDR.

\subsection{Trending tools}

A variety of post-launch trending tools were developed to track short-term and long-term detector stability and instrument telemetry points. Trending the detector scan-toscan raw $\mathrm{DN}$ is a fundamental method to check for abnormal behavior of the detector response, especially useful for the TEB because of the scan-to-scan calibration algorithm. The DN trending tool will be widely used postlaunch to examine detector response whenever there is a change in the detector's gain or detector signal-to-noise (SNR) level. The trending results of this analysis tool can help assess the sensor's health and data quality. As for detector noise characterization, similar tools will be used to trend detector short-term and long-term stability in terms of SNR for the RSB and noise equivalent difference in temperature (NEdT) for the TEB. These trending results help monitor any change of the VIIRS detectors in terms of gain, noise, and out-of-family behaviors. The outcome of this analysis will assist the decision of recommending SDR LUT updates. The detector trending tools are band, detector, and HAM side dependent.
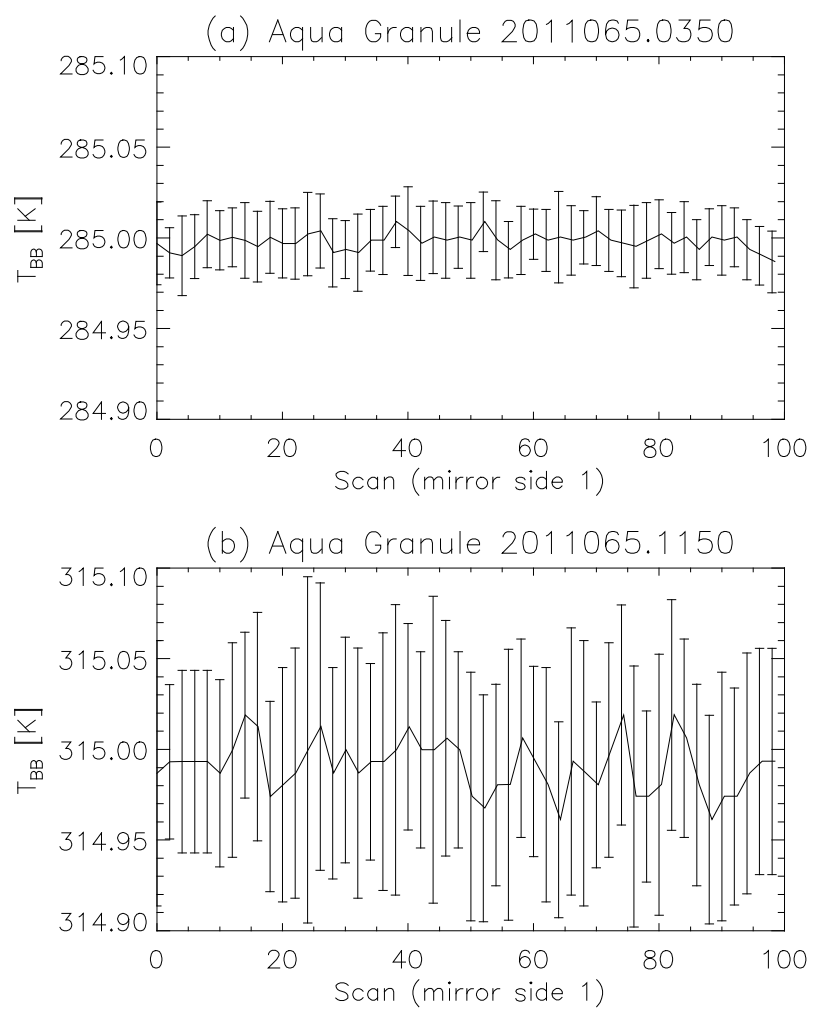

Fig. 2 Aqua MODIS blackbody temperature profiles during the WUCD activity in February 2011.

The VIIRS post-launch characterization often requires information derived from measurements of many temperature telemetries, including those measuring the OBC-BB, the cavity, the HAM, the focal planes, and the electronic circuit card assemblies. Major temperature telemetry points related to VIIRS SDR radiometric calibration and characterization have been selected and 
applied to the telemetry trending tool. Some of the temperatures, such as those from the OBC-BB and HAM, are actually used in the radiometric calibration algorithm of the SDR operational code. Figure 2 shows trending plots of Aqua MODIS blackbody temperature profiles at two fixed temperature plateaus, controlled at $285 \mathrm{~K}$ and $315 \mathrm{~K}$, during a BB warm-up and cool-down (WUCD) cycle. These charts demonstrate the use of an NICSE developed tool to track $\mathrm{BB}$ temperature scan by scan, which is needed in the VIIRS SDR (equivalent to MODIS Level-1B data) TEB radiometric calibration. There are twelve temperature thermistors embedded in the MODIS BB while VIIRS only has six. Data shown here are the mean and the standard deviation error bar of six selected MODIS BB thermistors (out of twelve) at mirror side (MS) 1 during a 5-minute granule. Only half of the data are plotted for clarity. The standard deviations of six thermistors temperature are about $0.02 \mathrm{~K}$ and $0.06 \mathrm{~K}$ at $285 \mathrm{~K}$ and $315 \mathrm{~K}$ respectively. Based on the results from the VIIRS thermal vacuum test, the VIIRS BB performed better than MODIS BB in terms of thermal uniformity. During the VIIRS F1 sensor level testing at hot plateau, the standard deviations of BB thermistors at 283K and $315 \mathrm{~K}$ are $0.007 \mathrm{~K}$ and $0.004 \mathrm{~K}$, respectively. The periodic BB WUCD operations will provide useful data to assess and validate the non-linearity of the TEB detector response.

In September 2010, about one year prior to the scheduled NPP launch, NICSE has successfully completed initial development of all the tools and tested them by using MODIS proxy data as part of the NPP SDS requirements. Prior to NPP spacecraft launch, all the calibration tools will be updated and improved based on the latest VIIRS data format and calibration algorithms so that the VIIRS data can be handled correctly.

\section{FUTURE EFFORT AND SUMMARY}

The NICSE is currently engaged in two major activities: supporting NICST for assessment of VIIRS F1 pre-launch calibration testing and preparing for post-launch calibration activities. Following pre-launch spacecraft level calibration and test data analyses, the NICST efforts will be focused on sensor launch readiness support while NICSE will continue to support the same efforts, including development and validation of sensor SDR LUTs that are critical for the calibration during the initial phase of orbit operation. For the SDR validation support, NICSE works closely with NPP SDS Land PEATE on the development of Land PEATE version of SDR operational code (Ops code). NICSE has received, installed, and tested the latest SDR Ops code provided by Land PEATE. The Ops code includes calibration for geometric and radiometric modules.

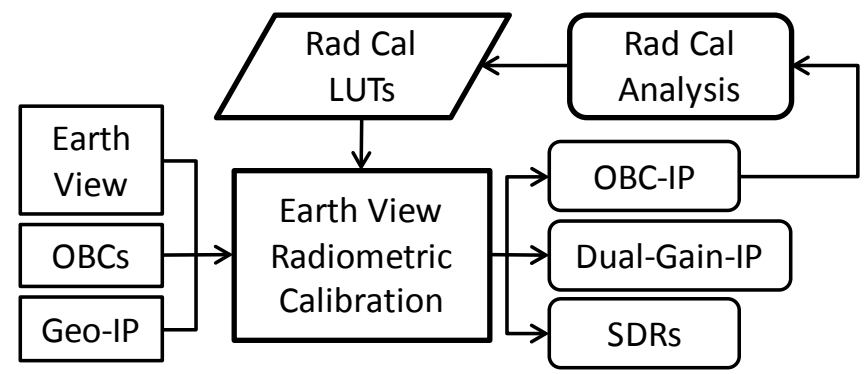

Fig. 3 VIIRS SDR Ops code radiometric calibration procedure.

Figure 3 shows only the radiometric part of the Ops code run on the NICSE system. The NICSE's major effort in support of VIIRS post-launch activities is involved with the radiometric calibration analysis for LUT improvement. Most of the tools described in this paper support such effort. With the ability to calibrate the detectors, update the SDR LUTs, and run the SDR Ops code to produce new SDRs, NICSE will be able to independently support VIIRS SDR validation.

The NASA NPP NICSE will continue to develop, test, and improve the planned data analysis and instrument monitoring tools. In addition to continuously making progress to complete all the scheduled tasks, NICSE has actively involved in support of extensive efforts to transition NPOESS program to the Joint Polar Satellite System (JPSS). With lessons learned from MODIS experiences and VIIRS pre-launch data analysis, NICSE has demonstrated its capability to improve calibration tools and develop new algorithms in the future to better monitor and understand onorbit changes in the sensor behavior.

\section{REFERENCES}

[1] R. Sikorski, K. Chiang, M. Nishihama, R. Wolfe, X. Xiong, and M. Schwaller, "An Overview of NASA NPP SDS-NICSE Activities on VIIRS SDR Assessment," Proceedings of IGARSS, 296-299, 2010.

[2] H. Oudrari, T. Schwarting, K. Chiang, J. McIntire, C. Pan, and X. Xiong, "A Methodology to Assess the Impact of Optical and Electronic Crosstalk in New Generation of Sensors Using Heritage Sensors,” Proceedings of IGARSS, 2691-2694, 2010.

[3] X. Xiong, K. Chiang, J. Esposito, B. Guenther, and W. Barnes, "MODIS On-orbit Calibration and Characterization," Metrologia 40, 89-92, 2003.

[4] K. Chiang, A. Wu, J. Sun, M. Schwaller, and X. Xiong, “Calibration Support for NPP SDR Assessment," Proceedings of SPIE - Earth Observing Systems XV, Vol. 7807, 78071A, 2010.

[5] J. Sun, X. Xiong, W. Barnes, and B. Guenther, "MODIS Reflective Solar Bands On-Orbit Lunar Calibration,” IEEE Trans. Geosci. Remote Sens., 45(7), 2383-2393, 2007.

[6] "VIIRS Radiometric Calibration Algorithm Theoretical Basis Document ATBD,” not published. 